much easier to let thinking follow the usual path indicated by a signal word, or let memory take over from it.

This avoidance is to be deplored. It can be overcome at least partly by good teaching. Students who have had sufficient practice both in thinking about new aspects of their subject and in eriticizing irrelevant responses may come to recognize more clearly what is relevant and to feel some confidence in their own ability to think independently. More independent study could also help, since thinking flexibly in a subject and perceiving most of its implications are made more easy by inereasing knowledge and work in it.

\title{
RESISTANCE OF GONOCOCCUS STRAINS TO PENICILLIN
}

GONOCOCCUS strains isolated in 1944 were found J to be highly sensitive to penicillin. From 1946 onwards, the existence of less sensitive strains were observed. Up to the end of 1955, doses of penicillin, twice to four times as great as those which were effective against the strains isolated in 1944, were enough to stop the development of the less sensitive strains isolated at the Statens Seruminstitut, Copenhagen. Then, from a case of gonorrhoea without complications, four strains were isolated which were found to be only one-twentieth as sensitive as the 1944 strains. This discovery and the fact that the incidence of gonorrhoea remained about the same in spite of penicillin therapy indicated the need for further research. This was carried out during 1957 and 1958 by Alico Reyn and M. W. Bentzon, who made a comparison of several hundred recently isolated gonococcus strains with a hundred strains isolated in 1944 (World Health Organization Chronicle, 14 ; No. 3. March 1960). This showed that, for inhibition, about 25 per cent of the strains sent to the laboratory for diagnosis in 1957 required doses of penicillin twenty times greater than had been needed for most strains originally.

Twenty strains showing reduced sensitivity to penicillin were subcultured for $30-90$ days on a medium with no penicillin, without any increase in sensitivity being produced.

Both in 1957 and 1958, some 200 strains were tested for penicillin sensitivity by the tablet method, that is to say, by placing penicillin tablets in the culture medium and measuring the zone of inhibition around them. The zone diameters varied between 20 and
$50 \mathrm{~mm}$. In both years, about 39 per cent of the strains showed reduced sensitivity, that is to say, zone diameters of $34 \mathrm{~mm}$. or less. In 1958, however, the number of strains with zone diameters of $44 \mathrm{~mm}$. or more was significantly lower than in 1957, which seems to indicate that the selection (and induction) of the strains with a comparatively reduced penicillin sensitivity continues.

None of the 1957 strains was completely resistant to streptomycin, but two of the 1958 strains (from the same patient) were.

In both the 1957 and 1958 strains a significant positive correlation was observed between sensitivity to penicillin and sensitivity to streptomycin.

From June 1957 onwards, atypical gonococcus strains-most of them sensitive to penicillinappeared in the cultures. The growth of these strains on the routine media is poor; they require special conditions for culture and are able to ferment glucose only weakly or not at all. Serological examination showed them to be distinguished from normal strains by the frequent presence of anti-complementary antigens. The proportion of atypical strains rose to 40 per cent in January 1958 when the culture and fermentation media were changed. The change of culture medium had yet another effect: the inhibition zones of the atypical strains-and of these alone-were greater on ox-heart broth than on oxmeat broth. This phenomenon has not yet been explained. It is possible that the emergence of atypical strains is related to the mode of action of the penicillin.

\section{GLOBULAR CLUSTERS}

A STRONOMY has always suffered because of the uneven distribution of its personnel and equipment between the northern and southern hemispheres. Especially welcome, therefore, are the growing number of contributions from the Radeliffe Observatory at Pretoria. A series of three contributions by Dr. T. D. Kinman (Mon. Not. Roy. Astro. Soc., 119, $157,538,559 ; 1959)$ represents an important addition to our knowledge of globular clusters. A study of their motions in the Galaxy is of interest because these clusters are prototypes of Baade's Population II and are believed to contain the oldest stars in the Galaxy. Kinman has obtained the radial velocities of thirty southern globular clusters, for sixteen of which no previous determinations of velocities existed; velocities are now available for seventy clusters in all, more than two-thirds of the known clusters in our Galaxy. The effect of the metal/hydrogen ratio on the strengths of the spectral lines of metallic elements in the clusters was studied by Kinman. He found that this ratio varies from
0.1 to less than 0.01 for different clusters. He also found that clusters of the earliest spectral type, the stars of which have a very low abundance of metal, form an extended spherical distribution with little concentration in the galactic plane, while those of latest type, the stars of which have the highest abundance of metal, show a strong concentration in the galactic plane. Clusters of intermediate tvpe show an intermediate distribution.

The motion of the Sun relative to the globular clusters was determined; the apex of the solar motion does not deviate from the direction of the Sun's galactic rotation. The new analysis confirms the older conclusion that the orbits of the individual clusters in the Galaxy are highly eccentric. The mean angular momentum per unit mass of the cluster system is the same as the mean for the rest of the Galaxy. This is in accord with the hypothesis that the clusters were formed in a spherical gas cloud which was in the process of contracting to form the galactic disk. 